\title{
Liposome encapsulated luteolin showed enhanced antitumor efficacy to colorectal carcinoma
}

\author{
GUIXIA WU $^{1 *}$, JING LI $^{1 *}$, JINQIAO YUE $^{2}$, SHUYING ZHANG $^{3}$ and KUREXI YUNUSI ${ }^{4}$ \\ ${ }^{1}$ Department of Physiology, Xinjiang Medical University, Urumqi, Xinjiang 830011; ${ }^{2}$ Department of \\ Medical Examination, Changji Vocational and Technical College, Changji, Xinjiang 830000; ${ }^{3}$ Department of \\ Gynaecology and Obstetrics, The 474th Hospital of People's Liberation Army of China; ${ }^{4}$ Department of \\ Biochemistry, Xinjiang Medical University, Urumqi, Xinjiang 830011, P.R. China
}

Received April 7, 2017; Accepted November 17, 2017

DOI: $10.3892 / \mathrm{mmr} .2017 .8185$

\begin{abstract}
Luteolin is a falconoid compound that is present in various types of plants and possesses remarkable potential as a chemopreventive agent. However, the poor aqueous solubility of luteolin limits its clinical application. In the present study, an approach towards chemoprevention was explored using liposomes to deliver luteolin, and the antitumor efficacy was investigated in colorectal carcinoma. The present findings demonstrated that luteolin was efficiently encapsulated into liposomes with an encapsulation efficiency as high as $90 \%$. The particle size of the liposomal luteolin (Lipo-Lut) and $\zeta$-potential were optimized. In vitro studies demonstrated that, Lipo-Lut had a significant inhibitory effect on the growth on the CT26 colorectal carcinoma cell line compared with free luteolin (Free-Lut). The in vivo study indicated that Lipo-Lut could achieve superior antitumor effects against CT26 tumor compared with luteolin alone. The present results suggested that liposome delivery of luteolin improved solubility, bioavailability and may have potential applications in chemoprevention in clinical settings.
\end{abstract}

\section{Introduction}

Colorectal carcinoma (CRC) is the third most common form of cancer in the world, and the rectum exhibits common internal malignancies (1). In China, CRC ranks 5th among

Correspondence to: Professor Kurexi Yunusi, Department of Biochemistry, Xinjiang Medical University, 393 Xinyi Road, Urumqi, Xinjiang 830011, P.R. China

E-mail: kuraxi8824@sina.com

*Contributed equally

Abbreviations: Lut, luteolin; NS, normal saline; Lipo-Lut, liposomal luteolin; Free-Lut, free luteolin; EM-Lipo, empty liposome

Key words: colorectal carcinoma, chemoprevention, luteolin, liposome, antitumor activity cancer deaths, with increasing the incidence annually (2). Although there are treatment options including radiotherapeutic, chemotherapeutic regimens and surgical regimens available for the clinical management of CRC, outcomes of such strategies are limited by associated high probability of cancer recurrence, obvious toxicity on the human body, affecting neurotoxicity, gastrointestinal reaction, kidney failure, and cardiotoxicity $(3,4)$. Chemoprevention is a strategy that was first proposed by Sporn et al (5). It is a way to reverse, suppress, or prevent molecular or histologic premalignant lesions from progressing to invasive cancer by using natural or synthetic agents (6). To date, many food/plant derived chemopreventive agents that exhibit strong efficacy against various cancers in vitro and preclinical models have been identified (7-10). Colorectal carcinoma is a rationale cancer to target for chemoprevention studies due to high incidence of pre-neoplastic lesions and cancerous tumors (11). An ideal chemopreventive compound should be nontoxic, potent, highly effective, less expensive, and easily available (4).

Luteolin (3',4',5,7-tetrahydroxyflavone), a flavonoid polyphenolic compound found in many plant types such as fruits, vegetables, and medicinal herbs. It has been shown biological activities, such as anti-inflammatory, anti-allergy, and anticancer activities (12-14). Recent studies have reported the anticancer effects of luteolin against lung cancer, head and neck cancer, prostate, breast, colon, liver, cervical, and skin cancer was associated with inducing apoptosis, suppressing metastasis, and angiogenesis (15-22). These results of above studies warrant the further evaluation of the chemopreventive potential of luteolin in human subjects (4). However, it has very low bioavailability after oral administration and it is very difficult to make intravenous or intraperitoneal administration because of its poor aqueous solubility. Therefore, there is a clear need to increase its potential in clinical application (23).

During the past few years, liposomes have drawn much attention for cancer therapy because of longer blood circulation time, higher biocompatibility, excellent bioavailability, and higher tumor-specific delivery (24-27). Thus, in the present study, we investigated whether liposomes can be used as delivery system to improve the antitumor efficacy of luteolin in vitro and in vivo. We used CT26 cell and mouse tumor model to evaluate the activity of luteolin before and 
after encapsulating into liposome. Meanwhie, we established a liposome-formulated luteolin that is capable of effective suppressing tumor growth through inducing apoptosis and inhibiting angiogenesis. It is our hope that understanding of these mechanisms in detail may provide a basis for novel targeting strategies for cancers.

\section{Materials and methods}

Liposome preparation. Lipo-Lut formulations were prepared by the thin film hydration method. Briefly, the mixtures of luteolin/cholesterol/lecithin (Sigma-Aldrich; Merck KGaA, Darmstadt, Germany) in 1:2:7 weight ratios were dissolved in ethanol and were transferred into a round bottom flask. The flask was then connected to a rotary evaporator at $50 \mathrm{rpm}$ and water bath with temperature maintained at $40^{\circ} \mathrm{C}$. Vacuum was applied to the flask to evaporate the ethanol and form a homogeneous lipid film on the flask wall. Then the lipid film was then hydrated in normal saline by rotating the flask at $37^{\circ} \mathrm{C}$ until the lipid film was completely hydrated. At last the luteolin liposome was sonicated with 50 watts of power for $10 \mathrm{~min}$. The empty liposome without luteolin was prepared in the same as the Lipo-Lut.

Size distribution and $\zeta$-potential. The mean particle sizes and $\zeta$-potential of the obtained liposomes were measured by photon correlation spectroscopy using Malvern Zeta sizer $3000 \mathrm{HS}$ (Malvern Instruments, Malvern, UK) at $25^{\circ} \mathrm{C}$. The form feature of the liposomes was determined by transmission electron microscope (TEM) (H-600; Hitachi, Tokyo, Japan) using a negative staining method with $1 \%$ sodium phosphotungstate solution for $2 \mathrm{~min}$ at room temperature.

Drug loading and encapsulation efficiency. The prepared liposomes were solubilized in methanol [liposomes: methanol=1:9, volume/volume (v/v)]. After using a cyclomixer to completely extract the drug from lipid to methanol, the drug content was analyzed by an Agilent 1100 Series HPLC System (Agilent Technologies, Santa Clara, CA, USA). An octadecylsilyl column (4.6x250.0 mm, $5 \mu \mathrm{m})$ was used for the analysis. The mobile phase was a mixture of acetonitrile and $0.1 \%$ formic acid $(30: 70, \mathrm{v} / \mathrm{v})$, and the flow rate was $0.5 \mathrm{ml} / \mathrm{min}$. The UV detection wavelength of $350 \mathrm{~nm}$ was used, the column temperature was $35^{\circ} \mathrm{C}$. The amount of soluble unencapsulated drug was measured by ultrafiltration using centrifugal filter tubes with a molecular weight cut-off of $300 \mathrm{kDa}$ (Millipore, Carrigtwohill, Ireland). Drug loading and encapsulation efficiency were calculated using following equations 1 and 2, respectively. The data were obtained using three different batches of liposome preparations.

$$
\begin{gathered}
\text { Drug loading (\%) }=\frac{C_{\mathrm{s}}}{C_{\text {lipid }}} \times 100 \% \\
\text { Encapsulation efficiency }(\%)=\frac{C_{\mathrm{s}}}{C_{\text {total }}} \times 100 \%
\end{gathered}
$$

Where $\mathrm{C}_{\mathrm{s}}$ represents drug mass in liposome, $\mathrm{C}_{\text {lipid }}$ represents total liposome mass, and $\mathrm{C}_{\text {total }}$ represents total drug mass.

Drug release. The release of luteolin from liposomes in vitro was measured with a dialysis method. Lipo-Lut solution
$(0.5 \mathrm{ml})$ and Free-Lut $(0.5 \mathrm{ml})$ in dimethyl sulphoxide (DMSO) solution were placed in a dialysis bag (MW cut off: $3500 \mathrm{Da}$ ). After that, dialysis bag was placed in a $50 \mathrm{ml}$ PBS supplemented with $0.5 \%$ Tween-20 (PBST). This bottle was introduced in a shaking incubator with stirring speed of $100 \mathrm{rpm}$ at $37^{\circ} \mathrm{C}$. At specific time intervals (0, $30 \mathrm{~min}, 1,2,4,6,8,12,24,48$, $72,96,120 \mathrm{~h}$ ), samples were withdrawn and replaced with an equal volume of medium. The amount of luteolin released at each time-point was determined using HPLC, as described earlier. All assays were performed in triplicate.

To measure in vivo pharmacokinetics, BALB/c mice, weighting 18-20 g were randomly divided into two groups (six animals per time-point) for treatment with Free-Lut or Lipo-Lut at a dose of $50 \mathrm{mg} / \mathrm{kg}$ body weight via intravenous injection. After dosing, blood was immediately collected via cardiac puncture at 5, 15, 30, 45, 1, 2, 4, 6, 8, 10, 12, $24 \mathrm{~h}$ and centrifuged at $8,000 \mathrm{rpm}$ for $10 \mathrm{~min}$ to separate the plasma. The diphenhydramine was added to plasma samples as the internal standard, and acetonitrile as protein precipitator. The drug was then extracted from plasma samples and processed for HPLC analysis to determine luteolin levels.

MTT assay. The murine CT26 colorectal carcinoma cell line was obtained from the ATCC, and it was maintained in RPMI1640 (Sigma-Aldrich; Merck KGaA) supplemented with 10\% fetal bovine serum (Invitrogen Life Technologies, Carlsbad, CA, USA) in a $5 \% \mathrm{CO}_{2}$ incubator at $37^{\circ} \mathrm{C}$. The inhibition of luteolin to CT26 cell was determined by the MTT assay. Briefly, cells in the logarithmic growth phase $\left(3 \times 10^{3}\right.$ or $5 \times 10^{3} /$ well $)$ were placed in wells of a 96 -well plate at $37^{\circ} \mathrm{C}$ overnight. Cells were then treated with various concentrations of Free-Lut or Lipo-Lut and cultured for 24. After an additional $3 \mathrm{~h}$ of culture with $0.5 \mathrm{mg} / \mathrm{ml} \mathrm{MTT}$ at $37^{\circ} \mathrm{C}, 150 \mu \mathrm{l} \mathrm{DMSO}$ was added to each well to dissolve formazan crystals. The cells which received only the medium containing $150 \mu \mathrm{l}$ DMSO served as the control group. All of samples were analyzed using a microplate reader (Bio-Rad Laboratories, Inc., Hercules, CA, USA). The test was repeated three times. The cell viability was assessed as a percentage of the absorbance present in the drug-treated cells compared to that in the control cells.

Assessment of apoptosis. The apoptotic rate of CT26 cells were detected by flow cytometry using the Annexin V-fluorescein isothiocyanate (FITC) and propidium iodide (PI) (Santa Cruz Biotechnology, Inc., Santa Cruz, CA, USA). CT26 cells cultured in 6-well plates were treated with various concentrations of Free-Lut or Lipo-Lut, along with no drug treatment as control. After incubation for $24 \mathrm{~h}$ at $37^{\circ} \mathrm{C}$, cells were trypsinized, washed and resuspended in PBS. The cells were subsequently treated with Annexin V-FITC and PI in the dark for $15 \mathrm{~min}$. Flow cytometry analysis was performed with an EpicsXL Coulter flow cytometer (Beckman Coulter, Fullerton, CA, USA) and repeated thrice.

Mouse tumor model. Female BALB/c mice, 6 weeks old and weighting 18-20 g, was obtained from Beijing HFK Bioscience Co., Ltd. (Beijing, China). The mice were kept in specific pathogen free (SPF) conditions for 1 week prior to start of experimental procedures. All the animal experiments were evaluated and approved by the Animal and Ethics Review 

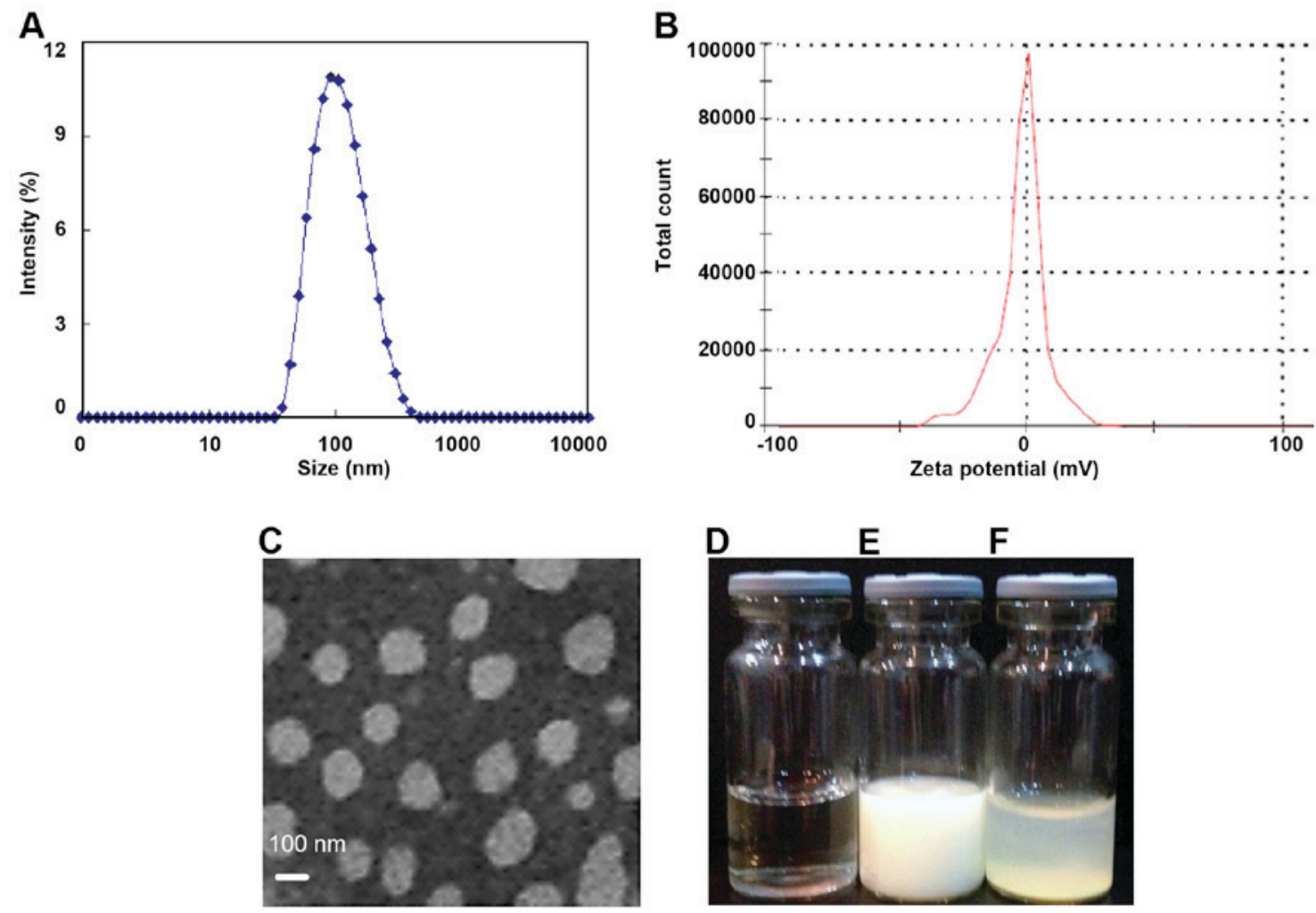

Figure 1. General characterization of liposomal luteolin. (A) Size distribution; (B) $\zeta$-potential spectrum; (C) TEM images of liposomal luteolin, the particles of liposomal luteolin had almost uniform, spherical shapes and were well dispersed; (D) normal saline; (E) water solution of liposomal luteolin (50 mg/ml); and $(\mathrm{F})$ luteolin in water $(50 \mathrm{mg} / \mathrm{ml})$. TEM, transmission electron microscope.

Committee of Xinjiang Medical University (Urumqi,Xinjiang). The murine tumor models were established by subcutaneous inoculation in the right flanks of female BALB/c mice with CT26 cells (1x10 $/$ mouse). The growth of the tumor was monitored, and tumor volumes were calculated from vernier calipers every 3 days, following the formula of $0.52 \mathrm{x}$ length $\mathrm{x}$ width ${ }^{2}$. The drug treatments were initiated when tumors had reached an average volume of $100 \mathrm{~mm}^{3}$, which occurred around day 7 post-cell inoculation. The luteolin dose administered to the mice was $50 \mathrm{mg} / \mathrm{kg}$. The mice were randomly assigned into four groups (six animals per group). Each group was respectively treated with normal saline (NS), empty liposome (EM-Lipo), Free-Lut, and Lipo-Lut via the tail vein every 2 days a total of five times. After the last treatment, the mice were sacrificed on the day 22 , and the tumors were excised, weighed and fixed in $10 \%$ neutral buffered formalin solution or frozen at $-80^{\circ} \mathrm{C}$.

Hematoxylin and eosin $(H \& E)$ staining and immunofluorescence staining. CT26 xenograft specimens were fixed in $4 \%$ paraformaldehyde for $12 \mathrm{~h}$ and embedded in paraffin. Sections (5- $\mu \mathrm{m}$ thick) were cut, dewaxed, rehydrated, and stained with H\&E. To observe the inhibitory effect on neovascularization, the frozen tissues were sectioned $(5 \mu \mathrm{m})$ and fixed in acetone. The tissues sections were incubated with monoclonal anti-CD31 antibody (Santa Cruz Biotechnology, Inc.) at $4^{\circ} \mathrm{C}$ overnight, flowing stained with a secondary goat antibody (FITC). The number of microvessels per high-power field was counted in sections. The immunofluorescence of Ki-67 expression in tumor was done as follows: Tissue sections were incubated with monoclonal anti-Ki67 antibody followed by incubation with FITC labelled goat anti-mouse secondary antibody (Santa Cruz Biotechnology, Inc.). Tissue apoptotic cells were detected with TUNEL Detection kit (Promega, Madison, WI, USA), according to the manufacturer's instructions. All the slides were evaluated by fluorescence microscope. Five areas were randomly selected from each slide for analysis.

Statistical analysis. The SPSS statistics 17.0 (SPSS, Inc., Chicago, IL, USA) were used for statistical analysis. Quantitative data were expressed as the mean \pm standard deviation of three independent experiments and analyzed by one-way ANOVA. Comparison between the groups was made by analyzing data with S-N-K method. A P-value $<0.05$ was considered to indicate a statistically significant difference.

\section{Results}

Characterization of Lipo-Lut. Our results demonstrated the successful application of the thin-film hydration method to formulate the water soluble Lipo-Lut. As shown in Fig. 1, dynamic light scattering results showed that the diameter of Lipo-Lut was around $105 \mathrm{~nm}$. The $\zeta$-potential of Lipo-Lut was $0.12 \mathrm{mv}$. The morphology of Lipo-Lut was observed using TEM, most Lipo-Lut was spherical and had a regular shape. Free-Lut appeared stratified in water, had apparent precipitation in the bottom of the bottle. Lipo-Lut can be stably suspended in water solution. Consequently, the drug loading and encapsulation efficiency were found to be 10 and $90 \%$, for the Lipo-Lut formulation. 

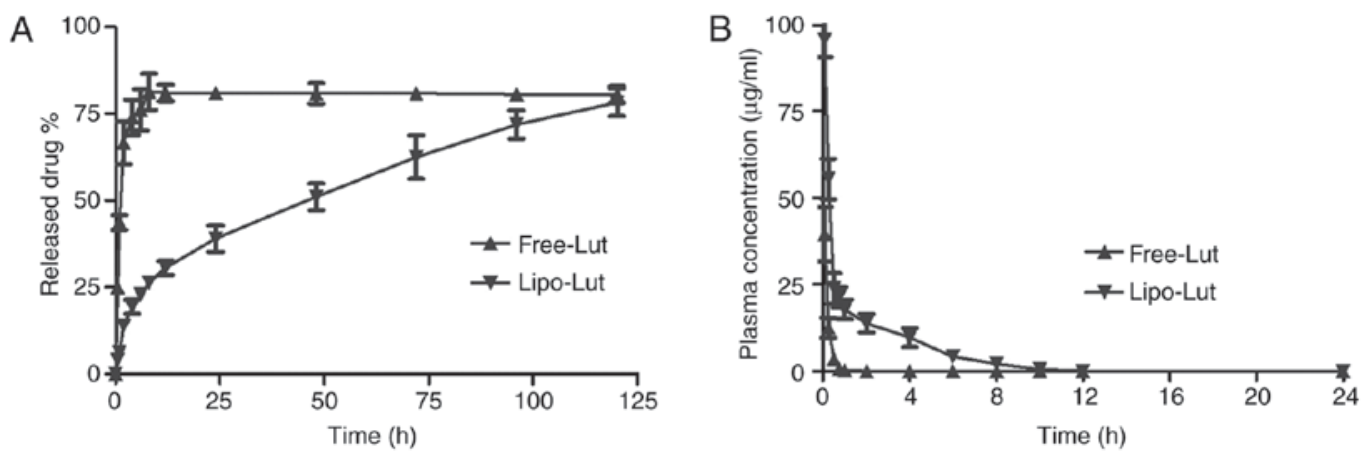

Figure 2. (A) In vitro release of Free-Lut or Lipo-Lut in $50 \mathrm{ml}$ phosphate-buffered saline; (B) plasma concentration-time curve of luteolin after administration of a single intravenous $50 \mathrm{mg} / \mathrm{kg}$ dose of Free-Lut or Lipo-Lut to mice. Data are expressed as mean \pm standard deviation, a representative result of three independent experiments is shown in each panel.

Drug release and Pharmacokinetics of Lipo-Lut. The in vitro release (Fig. 2A) results showed that the luteolin can be released slowly from the liposomes and then Free-Lut was released very quickly. The cumulative percentage release demonstrated that the amount of drug released from liposomes was gradually increased over time, and after $120 \mathrm{~h}$ there was an increase of over $80 \%$. The free drug exhibited high level $(80 \%)$ at $8 \mathrm{~h}$.

To assess whether the liposome improved bioavailability of poor water soluble drugs, the mean plasma concentration-time profiles of luteolin after intravenous administration of free and liposome drug were presented in Fig. 2B. Free-Lut was rapidly cleared and the plasma levels of luteolin were less than $50 \%$ of the injected dose within $5 \mathrm{~min}$ of injection. Compared to free drug, luteolin concentration in plasma was almost 10-fold higher for Lipo-Lut at $2 \mathrm{~h}$ after drug injection, a result that was most evident at time-points beyond $1 \mathrm{~h}$. The results demonstrated that liposomal encapsulation reduced drug elimination.

Lipo-Lut demonstrated better tumoricidal effect on colorectal cancer cells than the Free-Lut. Lipo-Lut and Free-Lut were evaluated for inhibition against CT26 cells using MTT assay. The results (Fig. 3) clearly established that Lipo-Lut exhibited potent inhibitory effect, as similar to Free-Lut. Further, both drugs showed dose-dependent inhibition of cell growth. After the incubation of the cells with Lipo-Lut or Free-Lut for $24 \mathrm{~h}$, the Lipo-Lut showed significantly higher inhibition compared to the Free-Lut at all the concentrations tested. In contrast, the empty liposomes did not show any toxicity to the cells (data not shown). These data indicated that Lipo-Lut inhibited tumor proliferation in vitro.

Next, we determined whether the inhibitory effect of Lipo-Lut involved the initiation of apoptosis. A flow cytometry analysis of Annexin V staining for phosphatidylserine, an early apoptosis marker, was performed for evaluation of apoptotic cells-after incubating CT26 cells with Lipo-Lut or Free-Lut at various concentrations. The cells were collected and stained with Annexin V-FITC and PI. When the cells undergoing apoptosis, the phosphatidylserine normally located in the inner leaflet of the cellular membrane translocates to the outer leaflet of the plasma membrane at the early stages of apoptosis, which can be labeled with Annexin V-FITC. Viable cells with intact

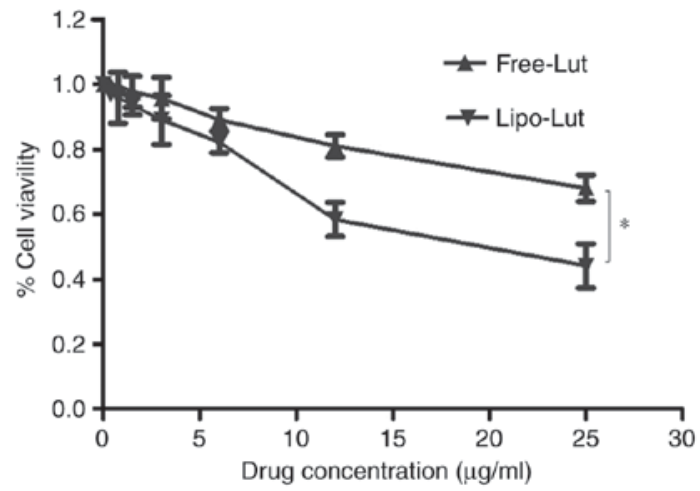

Figure 3. The effect of liposomal luteolin in CT26 cells viability. The CT26 cells were treated with different concentrations of Free-Lut or Lipo-Lut $(\mu \mathrm{g} / \mathrm{ml})$ for $24 \mathrm{~h}$. After this treatment, the cell viability was measured by MTT assay. The data are expressed as the mean \pm standard deviation of three independent experiments. ${ }^{*} \mathrm{P}<0.05$.

membranes exclude PI whereas the membranes of dead and damaged cells are permeable to PI. CT26 cells were divided into four groups, ie, necrotic cells (upper left quadrant, Annexin $\mathrm{V}^{-} / \mathrm{PI}^{+}$), healthy viable cells (lower left quadrant, Annexin $\mathrm{V}^{-} / \mathrm{PI}^{-}$), cells in the early apoptosis stage (lower right quadrant, Annexin $\mathrm{V}^{+} / \mathrm{PI}$ ), and cells that are in late apoptosis or already dead (upper right quadrant, Annexin $\mathrm{V}^{+} / \mathrm{PI}^{+}$). As shown in Fig. 4, more than 95\% of control CT26 cells (control) were viable whereas all the cells incubated with Lipo-Lut or Free-Lut displayed evidence of apoptosis. The percentage of early apoptotic cells and late apoptotic cells in Lipo-Lut or Free-Lut were showed dose dependence. Meanwhile, the extent of apoptosis in the Lipo-Lut group was significantly higher than Free-Lut group.

Lipo-Lut demonstrated better tumoricidal effect on the mouse tumor model than the Free-Lut. To validate antitumor efficiency of Lipo-Lut in vivo, we used a CT26 colorectal carcinoma graft model in BALB/c mice (Fig. 5). Fig. 5A displayed the tumor volume of each group during the 22-day treatment. Compared to the NS and EM-Lipo, the final tumor volume of mice treated with luteolin was notably reduced. The Lipo-Lut group exhibited the most significant inhibitory efficacy compared with the other groups. There was no significant difference between NS group and EM-Lipo group. At the end 

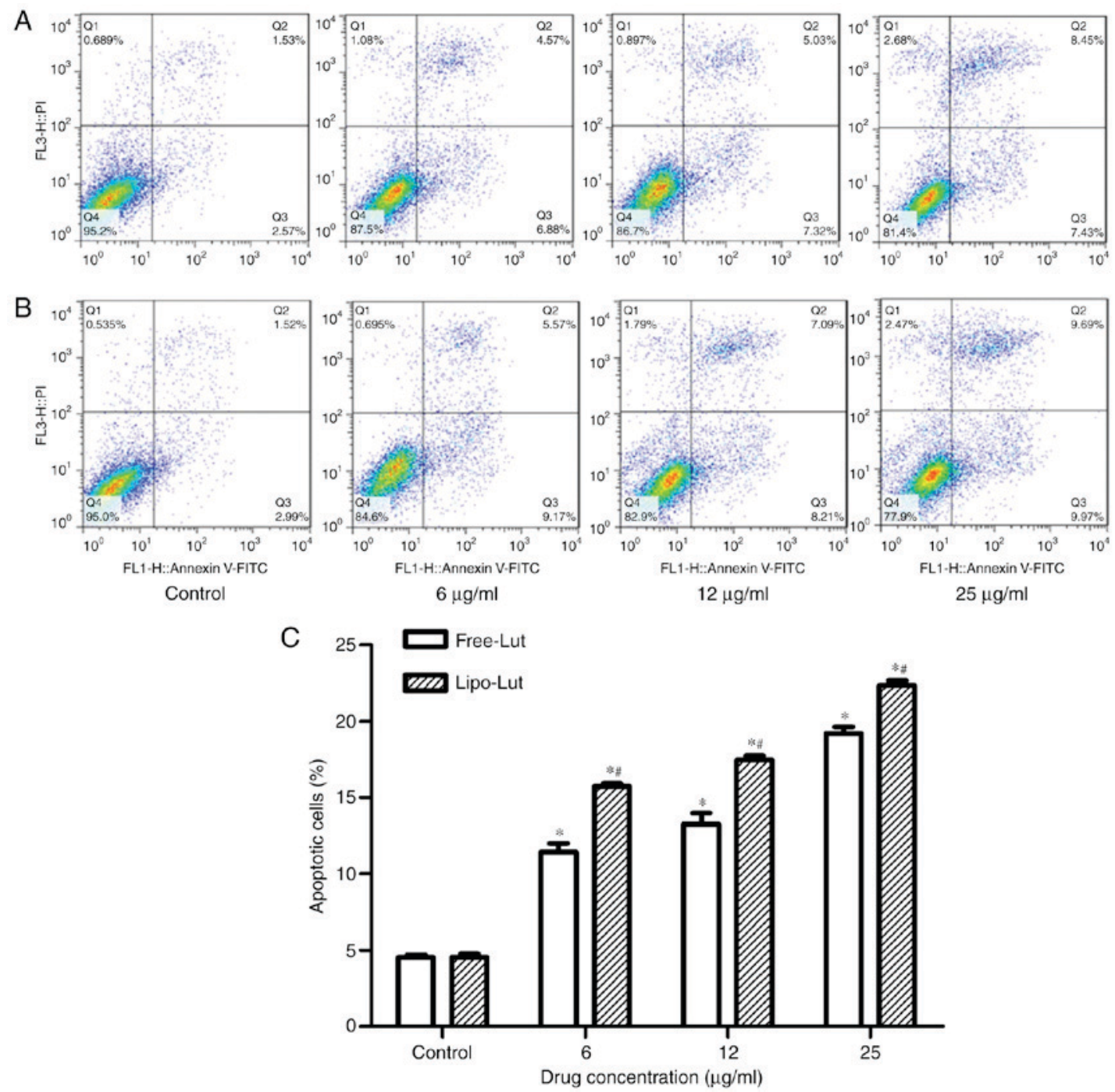

Figure 4. The apoptotic effect of liposomal luteolin on CT26 cells in vitro. CT26 cells were treated without (control) or with different concentrations of Free-Lut or Lipo-Lut and subjected to Annexin V and PI double staining. The apoptosis of the CT26 cells was detected by flow cytometry. (A) Free-Lut; (B) Lipo-Lut; (C) the representative results of apoptotic cells were shown. The percentage of apoptotic cells was determined from the fluorescence signal in excess over that obtained with negative (unlabeled) control cells. The data are expressed as the mean \pm standard deviation of three independent experiments. ${ }^{*} \mathrm{P}<0.05$ vs. control; ${ }^{*} \mathrm{P}<0.05$ vs. Free-Lut.

of the treatment, the tumor weights were obtained (Fig. 5D), the results were consistent with the tumor volumes at the end of the treatment. The smallest tumor weights were observed in the Lipo-Lut group among the four groups. These results indicated that the treatment of Lipo-Lut resulted in a robust efficacy in reducing tumor volume. Body weights were not significantly different in the four groups throughout the whole experiment (Fig. 5B).

Lipo-Lut induced apoptosis. To further investigated whether the in vivo antitumor effects of the Lipo-Lut were associated with enhanced induction of the apoptotic cells, TUNEL assay was applied. As shown in Fig. 6, a significant number of apoptotic cells appeared with green fluorescence in the tumor tissue of luteolin-treated mice. Treatment with Lipo-Lut clearly produced more pronounced apoptotic cells than treatment with the Free-Lut. These results suggested that Lipo-Lut inhibited tumor growth probably through induction of tumor cellular apoptosis.
Lipo-Lut inhibited tumor vascularization. We performed immunofluorescence analysis with anti-CD31 monoclonal antibody to observe the new vasculature content in frozen tumor sections (Fig. 7). As shown in Fig. 7D, CD31-positive endothelial cells in luteolin treated groups had weaker fluorescence than those of NS group and EM-Lipo group (Fig. 7A and B). The results from the determination of microvessel numbers (Fig. 7E) revealed that there were significantly less number of microvessels present in the Free-Lut group and Lipo-Lut group compared to the control group $(\mathrm{P}<0.01)$. In addition, Lipo-Lut group reduced number of microvessels more remarkably compared with Free-Lut groups $(\mathrm{P}<0.01)$. No significant difference was observed between NS group and EM-Lipo group $(\mathrm{P}>0.05)$.

Lipo-Lut decreased Ki-67 expression. The expression of $\mathrm{Ki}-67$ is strictly associated with tumor cell proliferation and growth, and is widely used in routine pathological investigation as a proliferation marker and a diagnosis tool (28). 

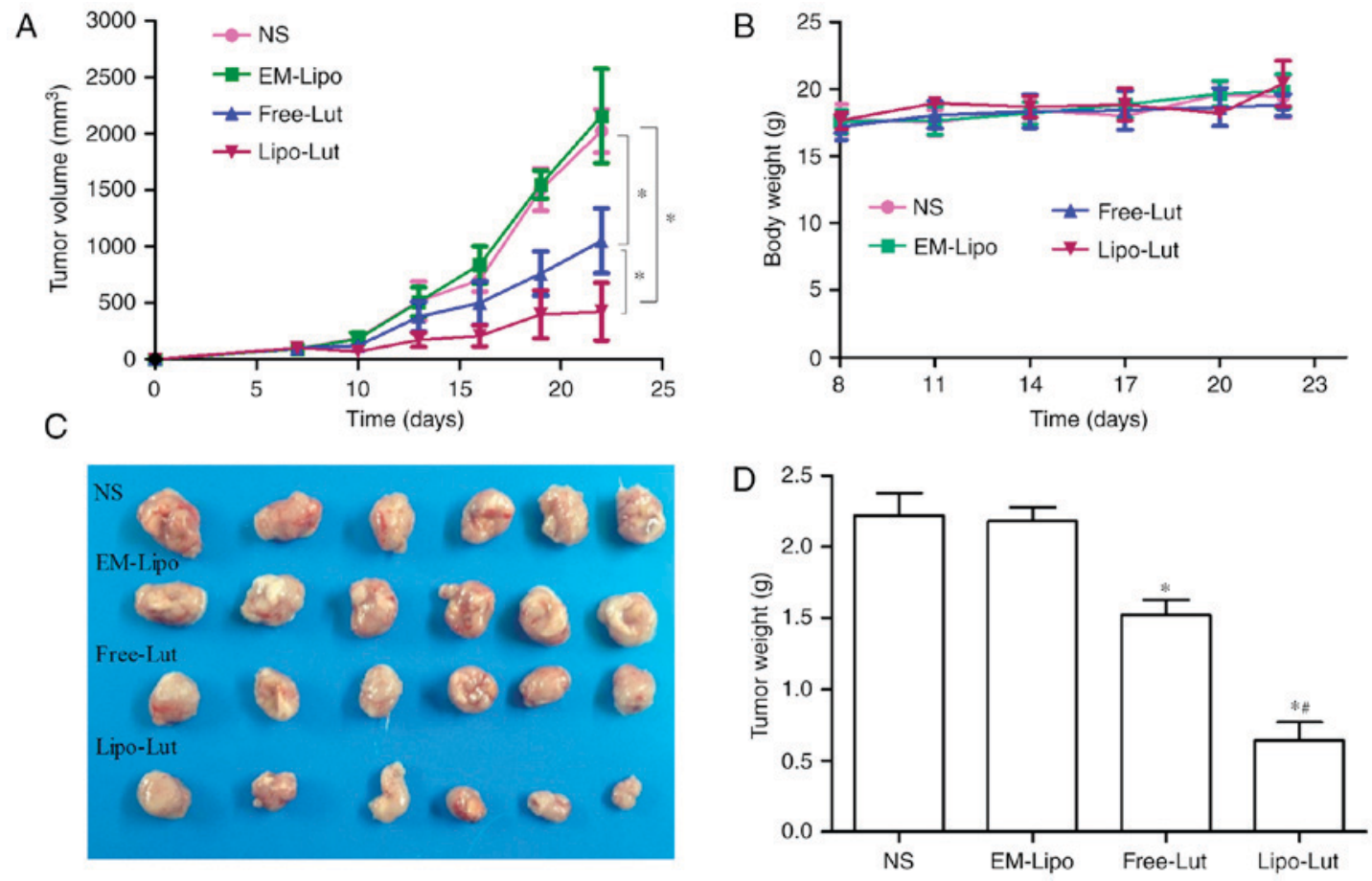

Figure 5. Antitumor efficacy of liposomal luteolin in vivo. Mice were given NS or EM-Lipo (as control), Free-Lut or Lipo-Lut of $50 \mathrm{mg} / \mathrm{kg}$ luteolin via the tail vein every 2 days for a total of five times. The tumor volumes and body weights were measured every 3 days following the day of administration. (A) Tumor volumes of CT26 tumor-bearing mice after treatment. (B) Body weight changes of mice during the efficacy test. (C) Representative mouse tumor from each group. (D) Tumor weights at the end of study. Data are presented as the mean \pm standard deviation of at least 6 different mice for each time-point. "P $<0.01$ vs. control; ${ }^{*} \mathrm{P}<0.01$ vs. Free-Lut.
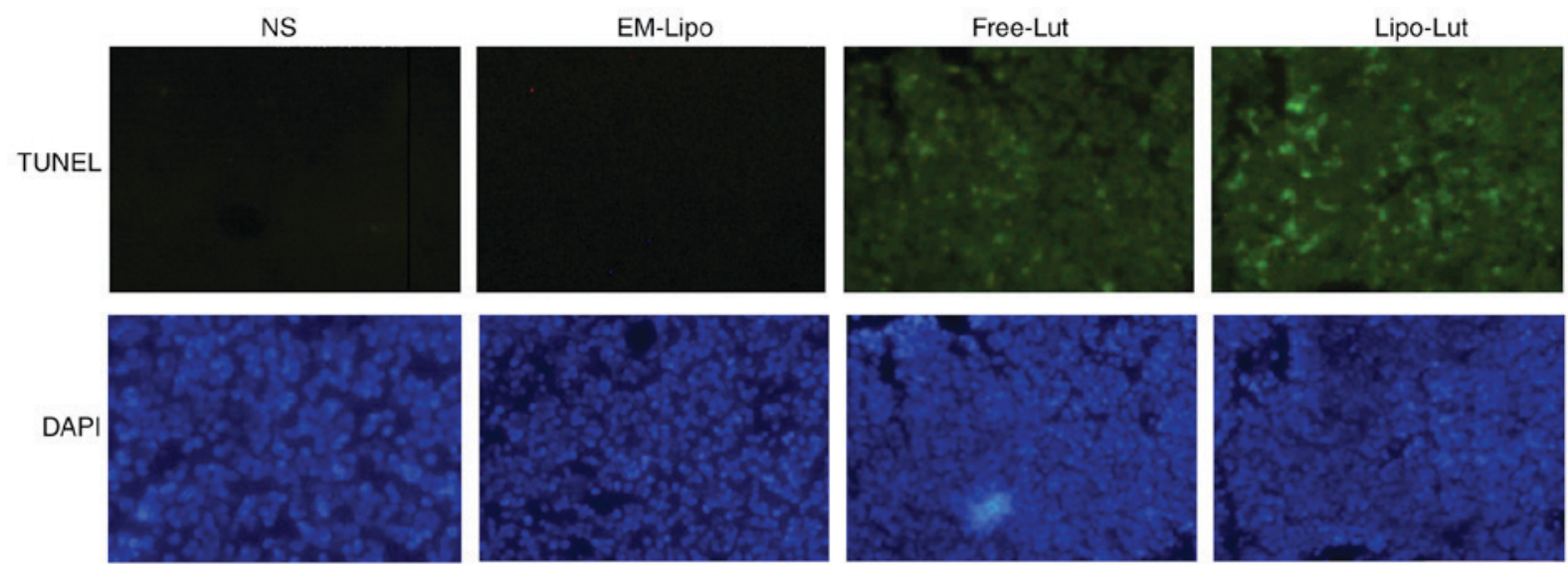

Figure 6. TUNEL detection of apoptosis. The frozen tumor tissues sections were visualized by fluorescence microscopy. The sections were subjected to TUNEL staining and DAPI. All original magnifications x200. DAPI, 4',6-diamidino-2-phenylindole.

Immunofluorescence examination of Ki-67 staining revealed a greater inhibition of proliferating cells in the Lipo-Lut-treated mice than other groups (Fig. 8).

\section{Discussion}

The present study demonstrated that liposome-encapsulated luteolin exerted stronger tumor growth-inhibition than free luteolin both in vitro and in vivo. Liposome have been widely used as potential drug delivery systems (DDS) for delivery of anti-cancer agents $(24,29)$. Over the past few decades, several liposome-encapsulated drugs have been approved for clinic applications. The best-known formulation is liposome-encapsulated doxorubicin, marketed as Doxil or Caelyx (30-32), which produced less cardiotoxicity than free doxorubicin while providing comparable antitumor activity $(33,34)$. In our study, we have prepared water soluble Lipo-Lut, sized $\sim 105 \mathrm{~nm}$. According to the cancer type, the size of the gaps between the endothelial cells of the tumor capillaries ranges from 100 to $780 \mathrm{~nm}$, as opposed to that in a typical normal endothelium of 5-10 nm $(35,36)$. Liposomes of about $100 \mathrm{~nm}$ in diameter have been demonstrated to be optimal for the delivery of anticancer drugs to tumors $(24,37)$, which readily translocate across the capillary endothelium. Liposomes are known to be safe and well tolerated delivery system. Many researchers demonstrated that the actions of 

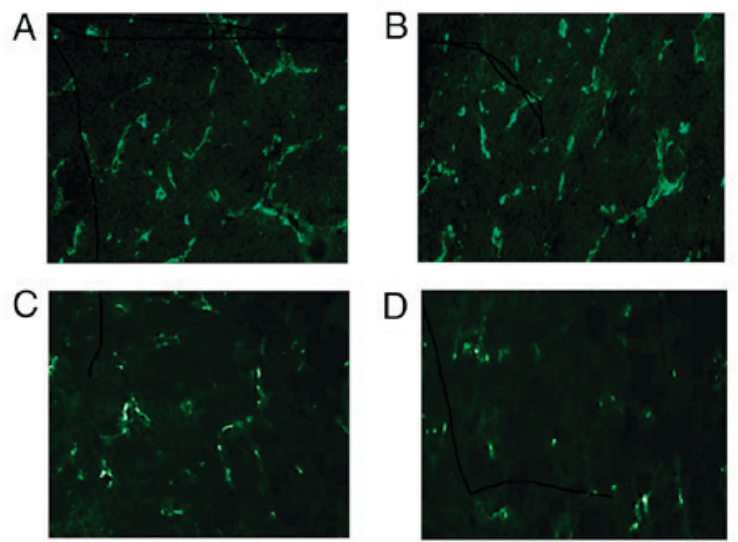

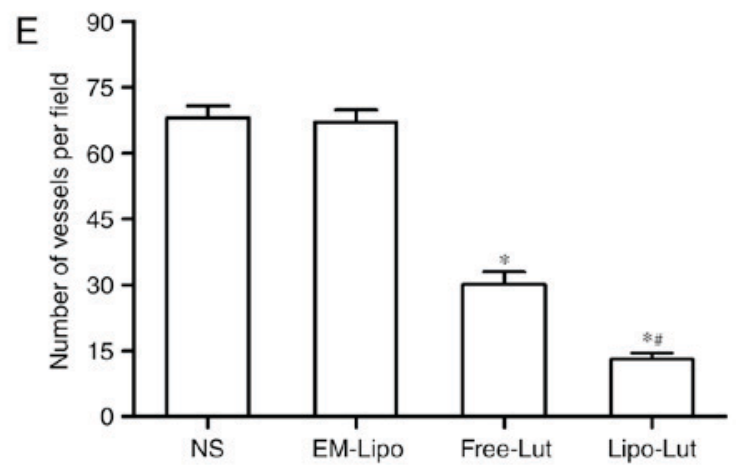

Figure 7. Inhibition of tumor angiogenesis. Frozen tumor tissue sections were stained for blood vessels by incubation with anti-CD31 Ab followed by incubation with secondary Ab conjugated with FITC and the tumor blood vessels were visualized by fluorescence microscopy. (A) NS; (B) EM-Lipo; (C) Free-Lut; (D) Lipo-Lut; and (E) the number of vessels per x100 field were counted, five fields per slide and at least three slides per group were detected. Data are presented as the mean \pm standard deviation. ${ }^{*} \mathrm{P}<0.01$ vs. control; ${ }^{\text {"}} \mathrm{P}<0.01$ vs. Free-Lut. FITC, fluorescein isothiocyanate.

H\&E

NS

EM-Lipo

Free-Lut

Lipo-Lut
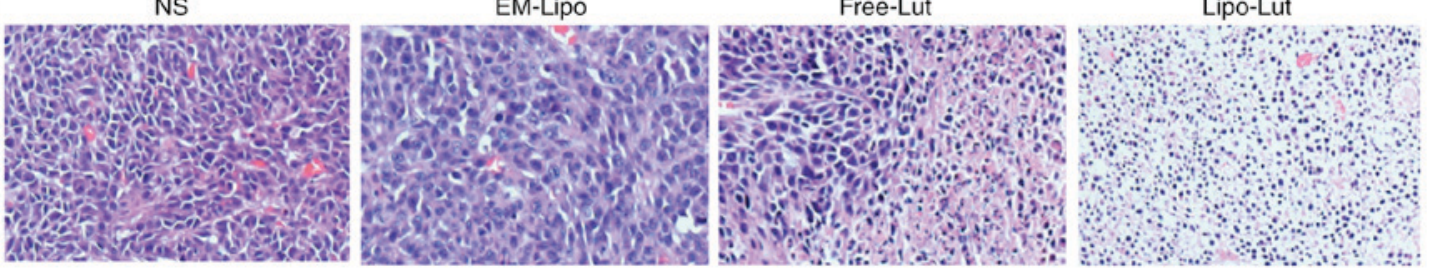

Ki 67
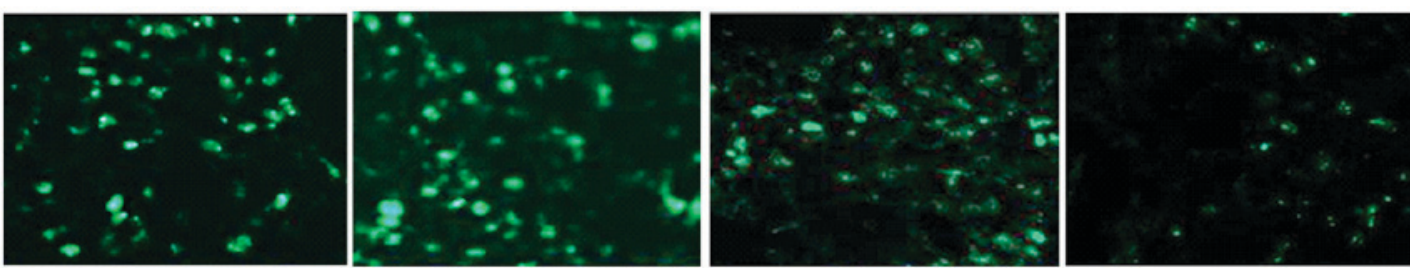

DAPI
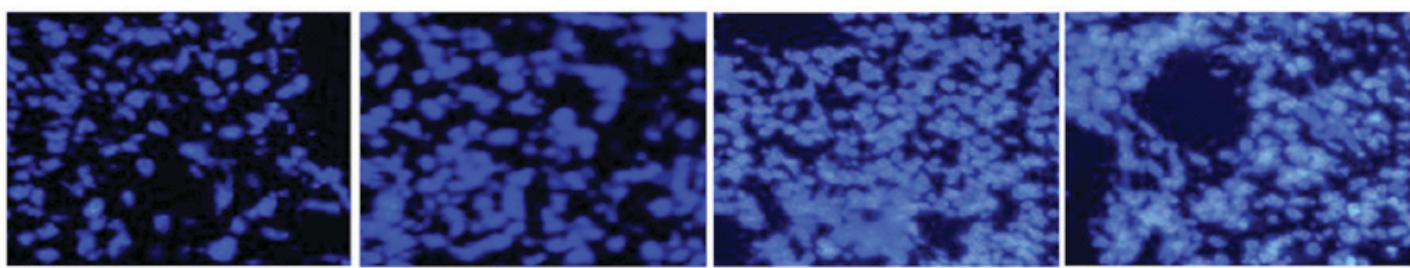

Figure 8. Inhibition of tumor proliferation. Representative H\&E-stained sections of tumor tissues were examined by light microscopy. Frozen tumor tissue sections were treated with anti-Ki-67 antibody followed by FITC-conjugated second Ab and DAPI. The stained sections were visualized by fluorescence microscopy. All original magnifications, x200. H\&E, hematoxylin and eosin; FITC, fluorescein isothiocyanate.

some poor water soluble drugs were evidently enhanced after they were encapsulated into liposome $(38,39)$. This study showed that Lipo-Lut could prolong the drug release. Meanwhile, the concentration in plasma-time profiles indicated that the bioavailability of Lipo-Lut has been improved.

In order to study the antitumor efficacy of Lipo-Lut in vitro, we initially compared the efficacy of Free-Lut and Lipo-Lut on CT26 cell growth inhibition by MTT assay and apoptosis by flow cytometry. We observed that Lipo-Lut exhibited more effectively than Free-Lut. This prompted us to further evaluate the antitumor activities of Lipo-Lut on CT26 cells grafts in BALB/c mice. The results suggested that luteolin encapsulated into liposome exerted stronger tumor growth-inhibiting effects, suppressed angiogenesis, increased apoptosis than Free-Lut. Angiogenesis plays a important role in the progress of tumor growth and invasion. The most essential angiogenic factors is vascular endothelial growth factor (VEGF). It have been reported that luteolin could significantly inhibit VEGF-stimulated endothelial cell proliferation, migration, invasion and tumor angiogenesis by targeting VEGF receptor 2-regulated AKT/ERK/mTOR/P70S69K/MMPs pathway, leading to the inhibition of tumor growth and tumor angiogenesis. A study from Norhaizan suggested that luteolin induced apoptosis in colon cancer by modulating the expressions of bax, Bcl-2 and caspase 3 in vitro and in vivo. On the other hand luteolin acted against DNA damage and activated DNA repair mechanism in Caco-2 colon cancer cells (40-42). Moreover, Owing to the leaky vasculature of the tumor tissue, 
these may provide a channel allowing liposome to more easily target tumor tissue (1). Meanwhile, solid tumors usually lack effective lymphatic drainage. All of these factors lead to the accumulation of liposome in the tumor microenvironment much more than they do in normal tissues $(43,44)$. Most likely because of a combination of these advantages, Lipo-Lut was a marked inhibition of tumor growth over time when compared to Free-Lut in a mouse tumor model. In fact, the present study also indicated that Nano-luteolin showed higher efficacy compared to Free-Lut against lung cancer and head and neck cancer (4). However, Lipo-Lut was unable to completely inhibit tumor growth. In order to gain better therapeutic efficacy, it is necessary to optimize liposomal formations and therapeutic scheme.

We prepared a novel antitumor agent using liposome as the delivery system to encapsulate luteolin. The Lipo-Lut inhibited activity of tumor growth more effectively than the Free-Lut in both CT26 cells and mouse tumor model of colorectal carcinoma. The mechanisms of action of Lipo-Lut appear multifaceted. Firstly, it could directly induce apoptosis of tumor cells. Secondly, tumor angiogenesis were reduced, blocking the nutrition supply into tumor tissue, which promoted apoptosis of tumor cells. Third, Lipo-Lut inhibited tumor proliferation. The results in this study could contribute to the development of chemotherapy for patients with colorectal carcinoma in future clinical applications.

\section{Acknowledgements}

The study was supported by the National Natural Science Foundation, People's Republic of China (grant no. 81660480) and Scientific Research Project of Xinjiang University (grant no. XJEDU2014I019).

\section{References}

1. Yang C, Liu HZ, Fu ZX and Lu WD: Oxaliplatin long-circulating liposomes improved therapeutic index of colorectal carcinoma. BMC Biotechnol 11: 21, 2011.

2. Nobili S, Checcacci D, Filippelli F, Del Buono S, Mazzocchi V, Mazzei T and Mini E: Bimonthly chemotherapy with oxaliplatin, irinotecan, infusional 5-fluorouracil/folinic acid in patients with metastatic colorectal cancer pretreated with irinotecan- or oxaliplatin-based chemotherapy. J Chemother 20: 622-631, 2008.

3. Chen AY and Chen YC: A review of the dietary flavonoid, kaempferol on human health and cancer chemoprevention. Food Chem 138: 2099-2107, 2013.

4. Majumdar D, Jung KH, Zhang H, Nannapaneni S, Wang X, Amin AR, Chen Z, Chen ZG and Shin DM: Luteolin nanoparticle in chemoprevention: In vitro and in vivo anticancer activity. Cancer Prev Res (Phila) 7: 65-73, 2014.

5. Sporn MB, Dunlop NM, Newton DL and Smith JM: Prevention of chemical carcinogenesis by vitamin A and its synthetic analogs (retinoids). Fed Proc 35: 1332-1338, 1976.

6. Madhunapantula SV and Robertson GP: Chemoprevention of melanoma. Adv Pharmacol 65: 361-398, 2012.

7. Mann CD, Neal CP, Garcea G, Manson MM, Dennison AR and Berry DP: Phytochemicals as potential chemopreventive and chemotherapeutic agents in hepatocarcinogenesis. Eur J Cancer Prev 18: 13-25, 2009.

8. Khor TO, Yu S and Kong AN: Dietary cancer chemopreventive agents-targeting inflammation and $\mathrm{Nrf} 2$ signaling pathway. Planta Med 74: 1540-1547, 2008.

9. Kaur M, Singh RP, Gu M, Agarwal R and Agarwal C: Grape seed extract inhibits in vitro and in vivo growth of human colorectal carcinoma cells. Clin Cancer Res 12: 6194-6202, 2006.
10. Kaur M, Velmurugan B, Tyagi A, Agarwal C, Singh RP and Agarwal R: Silibinin suppresses growth of human colorectal carcinoma SW480 cells in culture and xenograft through down-regulation of beta-catenin-dependent signaling. Neoplasia 12: 415-424, 2010.

11. Benbrook DM, Guruswamy S, Wang Y, Sun Z, Mohammed A, Zhang Y, Li Q and Rao CV: Chemoprevention of colon and small intestinal tumorigenesis in APC(min/+) mice by SHetA2 (NSC721689) without toxicity. Cancer Prev Res (Phila) 6: 908-916, 2013.

12. Yang MY, Wang CJ, Chen NF, Ho WH, Lu FJ and Tseng TH: Luteolin enhances paclitaxel-induced apoptosis in human breast cancer MDA-MB-231 cells by blocking STAT3. Chem Biol Interact 213: 60-68, 2014

13. Huang X, Dai S, Dai J, Xiao Y, Bai Y, Chen B and Zhou M: Luteolin decreases invasiveness, deactivates STAT3 signaling and reverses interleukin- 6 induced epithelial-mesenchymal transition and matrix metalloproteinase secretion of pancreatic cancer cells. Onco Targets Ther 8: 2989-3001, 2015.

14. Yan M, Liu Z, Yang H, Li C, Chen H, Liu Y, Zhao M and Zhu Y: Luteolin decreases the UVA-induced autophagy of human skin fibroblasts by scavenging ROS. Mol Med Rep 14: 1986-1992, 2016.

15. Lee HZ, Yang WH, Bao BY and Lo PL: Proteomic analysis reveals ATP-dependent steps and chaperones involvement in luteolin-induced lung cancer $\mathrm{CH} 27$ cell apoptosis. Eur J Pharmacol 642: 19-27, 2010.

16. Ong CS, Zhou J, Ong CN and Shen HM: Luteolin induces G1 arrest in human nasopharyngeal carcinoma cells via the Akt-GSK-33-cyclin D1 pathway. Cancer Lett 298: 167-175, 2010.

17. Chiu FL and Lin JK: Downregulation of androgen receptor expression by luteolin causes inhibition of cell proliferation and induction of apoptosis in human prostate cancer cells and xenografts. Prostate 68: 61-71, 2008

18. Cai X, Ye T, Liu C, Lu W, Lu M, Zhang J, Wang M and Cao P. Luteolin induced G2 phase cell cycle arrest and apoptosis on non-small cell lung cancer cells. Toxicol In Vitro 25: 1385-1391, 2011.

19. Balyan R, Kudugunti SK, Hamad HA, Yousef MS and Moridani MY: Bioactivation of luteolin by tyrosinase selectively inhibits glutathione S-transferase. Chem Biol Interact 240: 208-218, 2015.

20. Liu JF, Ma Y, Wang Y, Du ZY, Shen JK and Peng HL: Reduction of lipid accumulation in HepG2 cells by luteolin is associated with activation of AMPK and mitigation of oxidative stress. Phytother Res 25: 588-596, 2011.

21. Abdel Hadi L, Di Vito C, Marfia G, Ferraretto A, Tringali C, Viani P and Riboni L: Sphingosine kinase 2 and ceramide transport as key targets of the natural flavonoid luteolinto induce apoptosis in colon cancer cells. PLoS One 10: e0143384, 2015.

22. Wölfle U, Esser PR, Simon-Haarhaus B, Martin SF, Lademann J and Schempp CM: UVB-induced DNA damage, generation of reactive oxygen species and inflammation are effectively attenuated by the flavonoid luteolin in vitro and in vivo. Free Radic Biol Med 50: 1081-1093, 2011.

23. Liu Y, Wang L, Zhao Y, He M, Zhang X, Niu M and Feng N: Nanostructured lipid carriers versus microemulsions for delivery of the poorly water-soluble drug luteolin. Int J Pharm 476: $169-177,2014$.

24. Yuan Y, Zhao Y, Xin S, Wu N, Wen J, Li S, Chen L, Wei Y, Yang $\mathrm{H}$ and Lin S: A novel PEGylated liposome-encapsulated SANT75 suppresses tumor growth through inhibiting hedgehog signaling pathway. PLoS One 8: e60266, 2013.

25. Fetterly GJ, Grasela TH, Sherman JW, Dul JL, Grahn A, Lecomte D, Fiedler-Kelly J, Damjanov N, Fishman M and Kane MP: Pharmacokinetic/pharmacodynamic modeling and simulation of neutropenia during phase I development of liposome-entrapped paclitaxel. Clin Cancer Res 14: 5856-5863, 2008.

26. Gabizon A, Catane R, Uziely B, Kaufman B, Safra T, Cohen R, Martin F, Huang A and Barenholz Y: Prolonged circulation time and enhanced accumulation in malignant exudates of doxorubicin encapsulated in polyethylene-glycol coated liposomes. Cancer Res 54: 987-992, 1994.

27. Guo L, Fan L, Ren J, Pang Z, Ren Y, Li J, Wen Z, Qian Y, Zhang L and Ma H: Combination of TRAIL and actinomycin D liposomes enhances antitumor effect in non-small cell lung cancer. Int J Nanomedicine 7: 1449-1460, 2012. 
28. Li LT, Jiang G, Chen Q and Zheng JN: Ki67 is a promising molecular target in the diagnosis of cancer (Review). Mol Med Rep 11: 1566-1572, 2015.

29. Koudelka S, Turanek Knotigova P, Masek J, Prochazka L, Lukac R, Miller AD, Neuzil J and Turanek J: Liposomal delivery systems for anti-cancer analogues of vitamin E. J Control Release 207: 59-69, 2015.

30. Barenholz Y: Doxil ${ }^{\circledR}$ - the first FDA-approved nano-drug: Lessons learned. J Control Release 160: 117-134, 2012.

31. Schiffelers RM, Metselaar JM, Fens MH, Janssen AP, Molema G and Storm G: Liposome-encapsulated prednisolone phosphate inhibits growth of established tumors in mice. Neoplasia 7: $118-127,2005$

32. Gabizon A and Martin F: Polyethylene glycol-coated (pegylated) liposomal doxorubicin. Rationale for use in solid tumours Drugs 4 (Suppl 54): S15-S21, 1997.

33. Harris L, Batist G, Belt R, Rovira D, Navari R, Azarnia N, Welles L and Winer E; TLC D-99 Study Group: Liposome-encapsulated doxorubicin compared with conventional doxorubicin in a randomized multicenter trial as first-line therapy of metastatic breast carcinoma. Cancer 94: 25-36, 2002.

34. Hioki A, Wakasugi A, Kawano K, Hattori Y and Maitani Y: Development of an in vitro drug release assay of PEGylated liposome using bovine serum albumin and high temperature. Biol Pharm Bull 33: 1466-1470, 2010.

35. Deshpande PP, Biswas S and Torchilin VP: Current trends in the use of liposomes for tumor targeting. Nanomedicine (Lond) 8 : $1509-1528,2013$

36. Haley B and Frenkel E: Nanoparticles for drug delivery in cancer treatment. Urol Oncol 26: 57-64, 2008.

37. Cukierman E and Khan DR: The benefits and challenges associated with the use of drug delivery systems in cancer therapy. Biochem Pharmacol 80: 762-770, 2010.

38. Fan Y, Liu J, Wang D, Song X, Hu Y, Zhang C, Zhao X and Nguyen TL: The preparation optimization and immune effect of epimedium polysaccharide-propolis flavone liposome. Carbohydr Polym 94: 24-30, 2013.
39. Zhu Y, Wang M, Zhang J, Peng W, Firempong CK, Deng W, Wang Q, Wang S, Shi F, Yu J, et al: Improved oral bioavailability of capsaicin via liposomal nanoformulation: Preparation, in vitro drug release and pharmacokinetics in rats. Arch Pharm Res 38: 512-521, 2014.

40. Pandurangan AK and Esa NM: Luteolin, a bioflavonoid inhibits colorectal cancer through modulation of multiple signaling pathways: A review. Asian Pac J Cancer Prev 15: 5501-5508, 2014.

41. Wang LM, Xie KP, Huo HN, Shang F, Zou W and Xie MJ: Luteolin inhibits proliferation induced by IGF-1 pathway dependent ER $\alpha$ in human breast cancer MCF-7 cells. Asian Pac J Cancer Prev 13: 1431-1437, 2012.

42. Pratheeshkumar P, Son YO, Budhraja A, Wang X, Ding S, Wang L, Hitron A, Lee JC, Kim D, Divya SP, et al: Luteolin inhibits human prostate tumor growth by suppressing vascular endothelial growth factor receptor 2-mediated angiogenesis. PLoS One 7: e52279, 2012.

43. Matsumura Y and Maeda H: A new concept for macromolecular therapeutics in cancer chemotherapy: Mechanism of tumoritropic accumulation of proteins and the antitumor agent smancs. Cancer Res 46: 6387-6392, 1986.

44. Vasey PA, Kaye SB, Morrison R, Twelves C, Wilson P, Duncan R, Thomson AH, Murray LS, Hilditch TE, Murray T, et al: Phase I clinical and pharmacokinetic study of PK1 [ $N$-(2-hydroxypropyl) methacrylamide copolymer doxorubicin]: First member of a new class of chemotherapeutic agents-drug-polymer conjugates. Cancer research campaign phase I/II committee. Clin Cancer Res 5: 83-94, 1999.

This work is licensed under a Creative Commons Attribution-NonCommercial-NoDerivatives 4.0 International (CC BY-NC-ND 4.0) License. 